\title{
Erratum to: Investigation of the inhibition effect of ibuprofen triazole against mild steel corrosion in an acidic environment
}

\author{
M. K. Pavithra • T. V. Venkatesha • \\ M. K. Punith Kumar $\cdot$ K. Manjunatha
}

Published online: 7 January 2015

(C) Springer Science+Business Media Dordrecht 2015

\section{Erratum to: Res Chem Intermed \\ DOI 10.1007/s11164-014-1804-5}

The original article was accepted and published online in September 2014. However, we regret to hereby bring to your notice that there has been a mistake in the Fig. 1 and 6-9 in which the organic molecule is represented. The corrected version of the figures is providing in the erratum.

The online version of the original article can be found under doi:10.1007/s11164-014-1804-5.

M. K. Pavithra · T. V. Venkatesha $(\bowtie)$

Department of Chemistry, School of Chemical Sciences, Jnana Sahyadri Campus, Kuvempu

University, Shimoga 577451, Karnataka, India

e-mail: drtvvenkatesha@yahoo.co.uk

M. K. Punith Kumar

Department of Materials Engineering, Indian Institute of Science, Bangalore 560012, Karnataka, India

K. Manjunatha

Department of Chemistry, Mangalore University, Mangala Gangothri 574199, Karnataka, India 

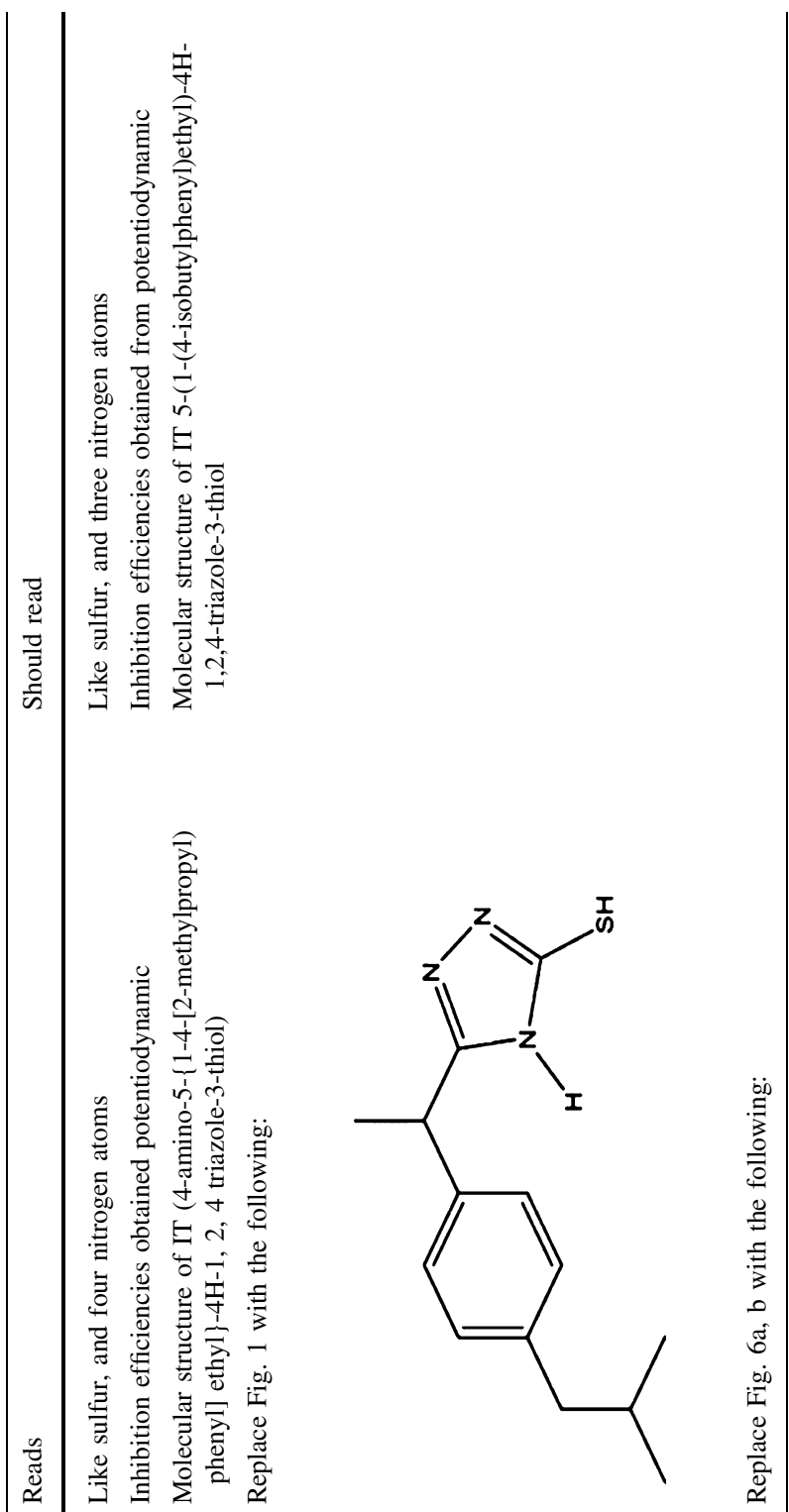

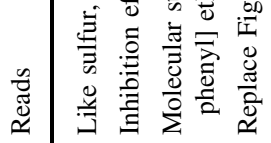

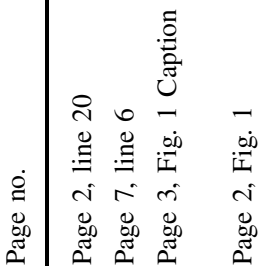




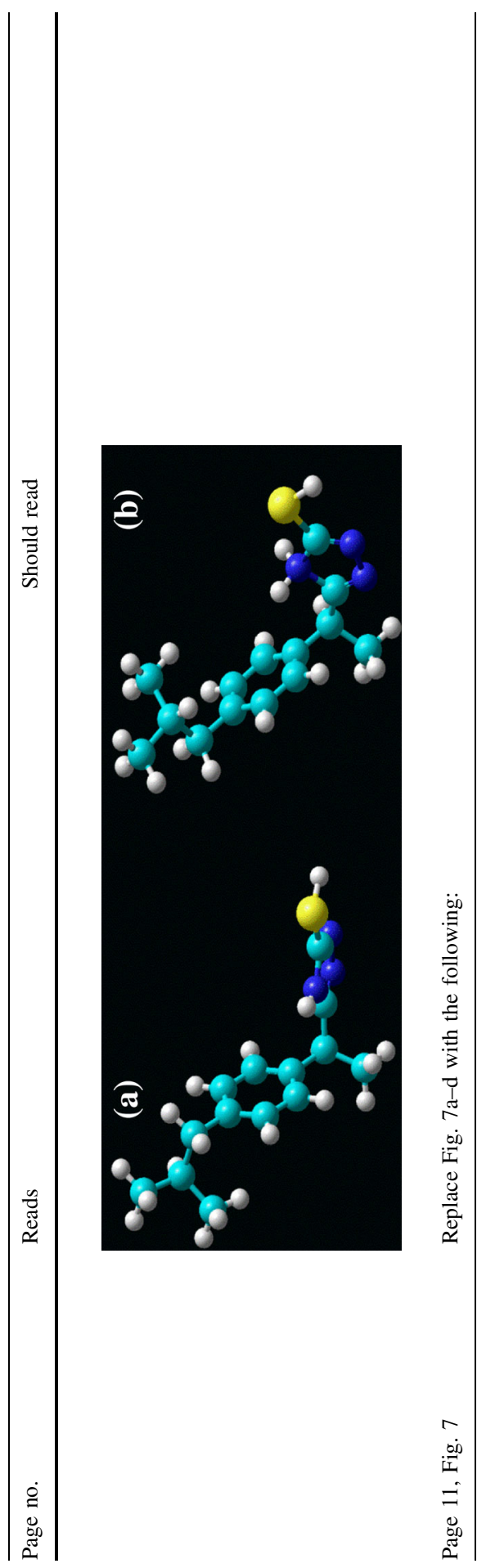


M. K. Pavithra et al.

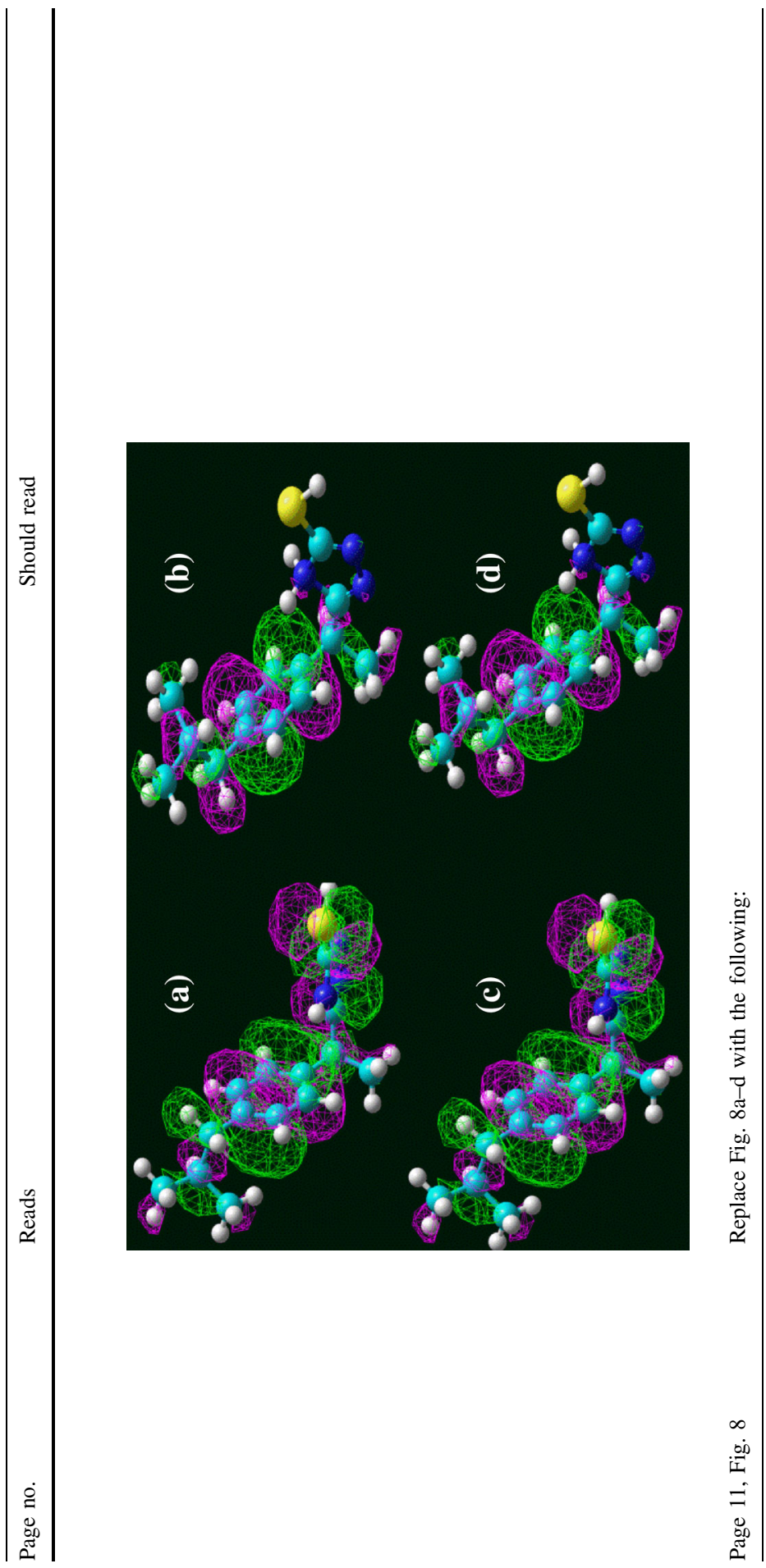




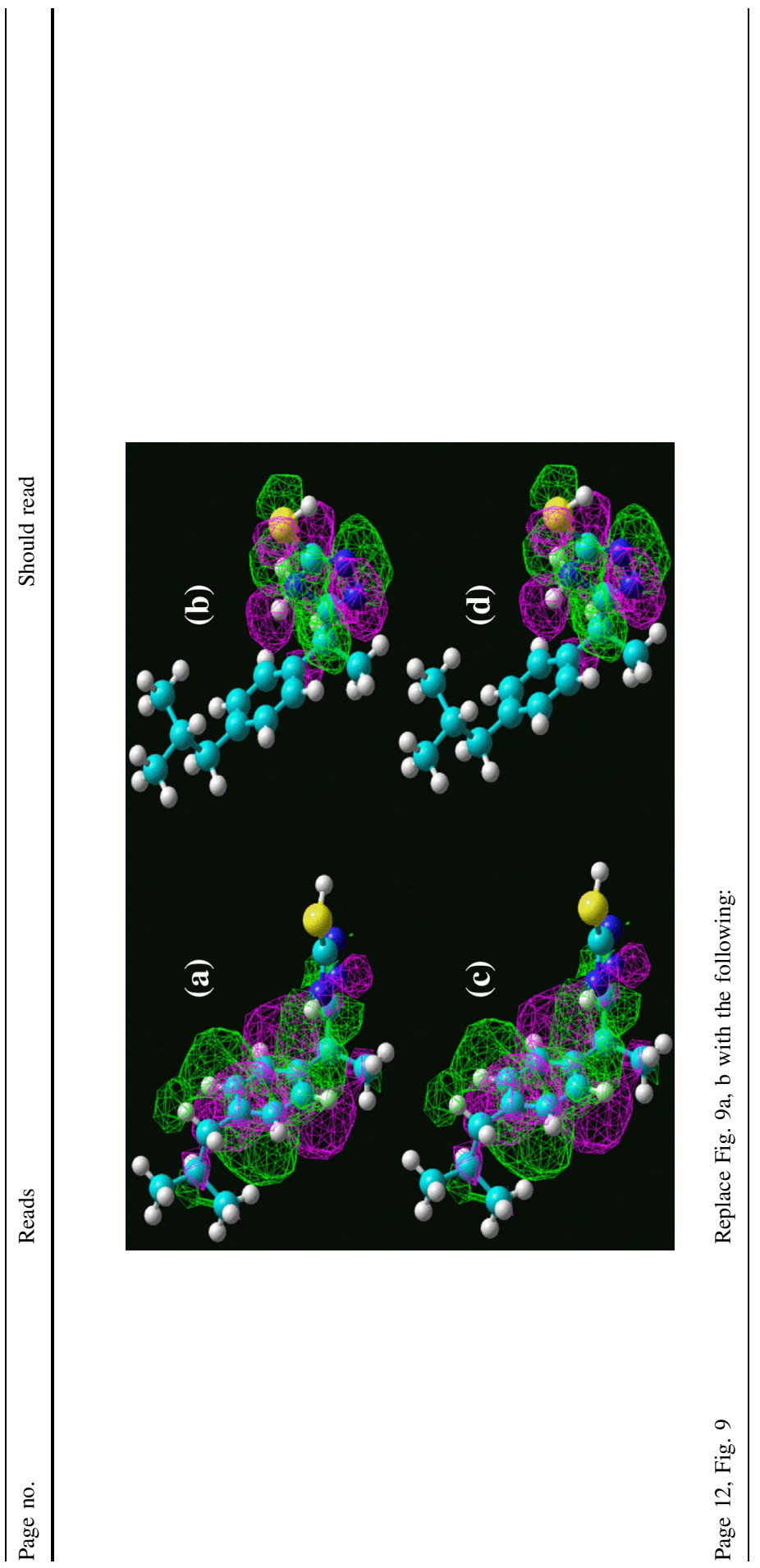




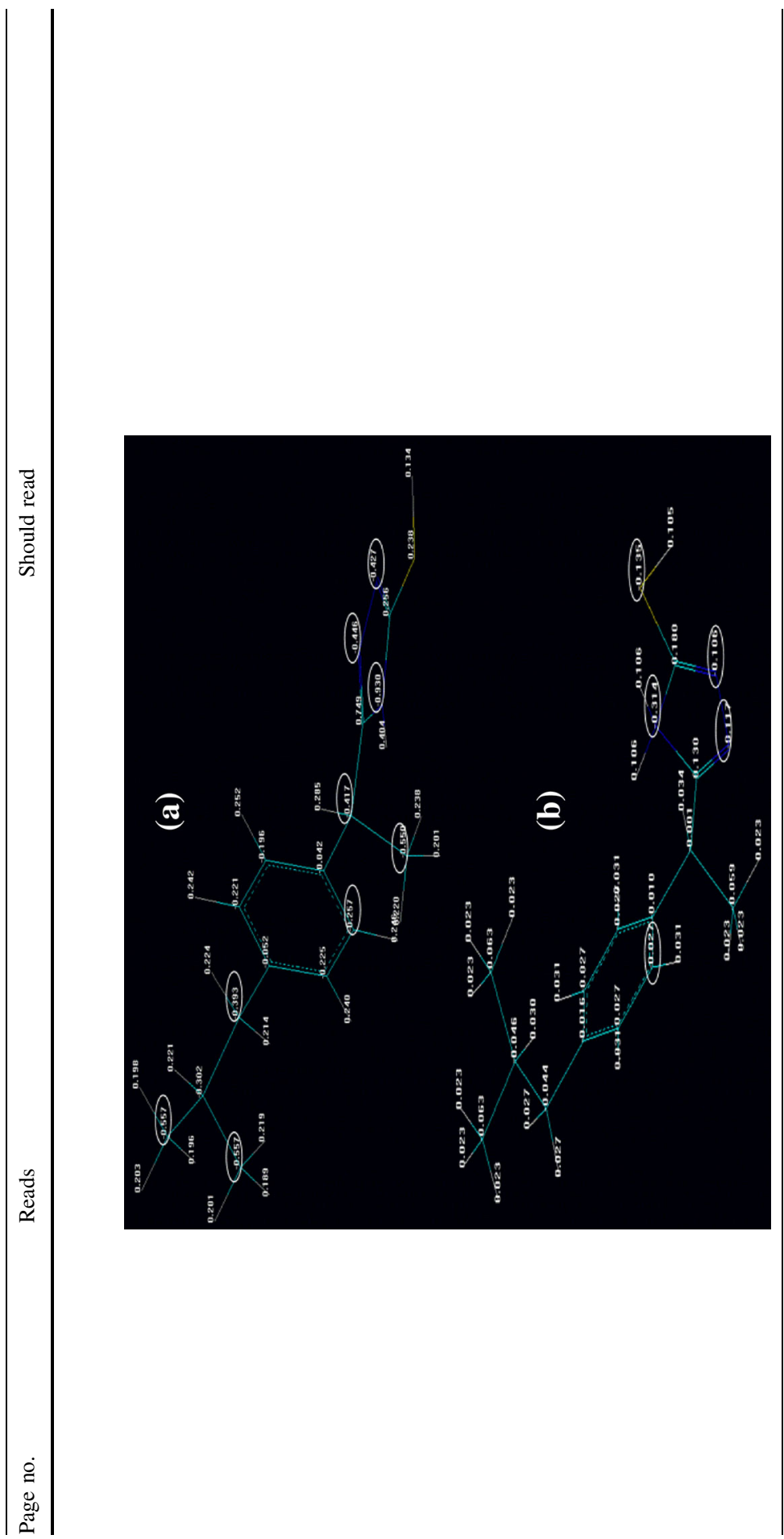


Page 10, Table 5

Replace Table 5 with the following table

\begin{tabular}{llllll}
\hline $\begin{array}{l}\text { Quantum chemical } \\
\text { parameters }\end{array}$ & \multicolumn{2}{l}{ Neutral species of IT molecule } & & \multicolumn{2}{l}{ Protonated species of IT molecule } \\
\cline { 2 - 3 } & $\alpha$ & & & $\alpha$ & $\beta$ \\
\hline $\mathrm{E}_{\text {HOMO }}(\mathrm{eV})$ & -9.08180 & -9.08184 & & -12.34152 & -12.34150 \\
$\mathrm{E}_{\mathrm{LUMO}}(\mathrm{eV})$ & 3.50684 & 3.50681 & & -2.21519 & -2.21515 \\
$\Delta E(\mathrm{eV})$ & 12.58864 & 12.58865 & & 14.55671 & 14.55665 \\
$\sigma$ & 0.14887 & 0.14887 & & 0.14750 & 0.14750 \\
$\Delta \mathrm{N}$ & 0.3146 & 0.3146 & & -0.0275 & -0.0275 \\
$\mu(\mathrm{D})$ & 5.223 & & & 297.3 & \\
Total energy & $-687,857$ & & & $-688,047$ & \\
$\quad(\mathrm{k} \mathrm{cal} \mathrm{mol}$ & & & & & \\
\hline
\end{tabular}

